\title{
Visual Analysis of Time-Motion in Basketball Games
}

\author{
Roberto Therón and Laura Casares \\ University of Salamanca
}

\begin{abstract}
This work aims to facilitate the task of basketball coaches, by means of the visualization and analysis of the players' movements in the court. This is possible thanks to the use of Global Positioning System (GPS) devices that generate data of the position of the player, almost in real time. The main objective of our proposal consists on the tracking, both statistics and kinematics, of a basketball player due to the physical activity developed during a match. The comparison of the data from several players or between two teams also will improve the performance and tactical capacity of players and trainers.

On one hand, the study of time-motion in sports is largely covered in the literature. On the other hand, the use of personal GPS devices for training purposes is a common practice. However, the design of interactive visualization tools that exploit the data stored in GPS devices during a match, thus enabling to perform its visual analysis, is still an open area. The work presented in this paper identifies the relevant aspects of the basketball game that are valuable for a coach in terms of team and individual performance analysis, and discusses the design and implementation of a tool that exploits the methods and techniques of a visual analytics approach.
\end{abstract}

\section{Introduction}

Today, one of the main features of a basketball coach is the visual memory. They often have to be able to remember plays and game situations for further analysis that will structure his team's tactics. Knowing what works and what fails becomes a great mental exercise whose source is the visual memory of the coach (also supported by game and training video recordings). Typically, to make this work, and only available after the game, the coach analyses the statistics of the two teams regarding converted baskets, personal fouls, free throws and the like.

Our proposal aims to facilitate the work of coaches and advance the study of basketball, offering a chance to see and analyse the movements that the players have previously made in the field of play, and their implications. In addition, we aim to provide both statistical and kinematic analysis of the data collected for each of players to facilitate monitoring over several physical exercises. The main data sources are individual wearable Global Positioning System (GPS) devices, that enable the collection of real time data related to the position of each player in the court during the whole match or training exercise.

Comparing data from several players or two teams are also aspects that will improve the performance of athletes through training.

The rest of the paper is organized as follows: Section 2 is devoted to a brief review of the current state of the research related to time-motion analysis in sports, its common 
methods and the opportunities that current technologies (GPS, visual analytics) have to offer to this field. Section 3 presents the requirements for basketball game analysis from a coach's viewpoint. Section 4 presents our visual analytics approach for basketball's time-motion analysis. Finally, Section 5 contains the conclusions derived from the work and exposes the future lines of research in which we are working now.

\section{Background}

Time-motion analysis (TMA) is a standard analytical procedure to determine the time and energy invested in an activity for a period of time [5]. Through this process the various patterns of movement involved in sport situations, such as speeds, durations or distances are collected and tallied [3]. Thus, we obtain valuable information on the use of energy systems, and on specific movement patterns of each sport [18]. In particular, TMA has been used in several disciplines, such as rugby [13] [11], football [6] [14][10], hockey [9][8], and basketball [12] [18].

The first aspect to take into account in TMA is the method used to acquire the data to be analysed. Once the data is stored and prepared, the analysis methodology itself has to be chosen. In the following we discuss the adequacy of GPS devices (as data source) and visual analytics (as analysis approach) that we have chosen to be used in our proposal.

\subsection{Methods for Time-Motion Analysis}

There are basically two ways to get data from TMA, video systems, which are by far the most used [3], and GPS devices. Regarding the former, using multiple cameras situated at a height and distance of variable pitch (in the case of basketball and other sports with small courts, like tennis, the height is between 1 and $5 \mathrm{~m}$ and the distance from the court between 2 and $10 \mathrm{~m}$ ).

The second approach has been less used so far as accuracy was quite low (in the case of positions, several meters of error). However, thanks to the development of differential GPS (d-GPS), using one or more additional reference stations on the ground (only satellite reference stations are used in traditional GPS), the accuracy increases considerably [22].

Furthermore, there is an ongoing effort to upgrade the Global Positioning System ${ }^{1}$ with new, advanced capabilities to meet growing military, civil, and commercial needs. The program is being implemented through a series of satellite acquisitions, including GPS Block IIR-M, GPS Block IIF, GPS Block III, and the Next-Generation Operational Control Segment (OCX). Three new signals have been designed for civilian use: L2C (the second civilian GPS signal, designed specifically to meet commercial needs; when combined with L1 C/A in a dual-frequency receiver, L2C enables ionospheric correction, a technique that boosts accuracy), L5 (the third civilian GPS signal, broadcast in a radio band reserved exclusively for aviation safety services), and L1C (the fourth civilian GPS signal, designed to enable interoperability between GPS and international

\footnotetext{
${ }^{1}$ http://www.space.commerce.gov/gps/modernization.shtml
} 
satellite navigation systems, i.e., Galileo). This establishes a panorama in which the research related to the design of tools that best exploit GPS devices is much needed, which is the case of our problem at hand.

Thus, GPS technology has been adapted in recent years to serve as a training tool, improving portability and generating useful data for the athlete (distance travelled, speed, etc.). But otherwise operate as a normal GPS, generating data at $1 \mathrm{~Hz}$ (once per second). Generally it has been used in sports' training covering long distances, such as walking or cycling. This breakthrough technology has also resulted in literature related to general human locomotion [19].

In both methods, in addition to the data that pertain to a typical TMA analysis, biological data, such as the athlete's heart rate, can also be monitored.

Currently the affordability of existing GPS wrist devices it is possible to have the 10 outfield players wearing one in a basketball game. These devices, despite having a margin of error, open the door for research into new technologies to improve the performance of top athletes, providing solutions for the particular case of basketball and for most team sports in which the precision errors are less noticeable.

\subsection{What Does Visual Analytics Have to Offer?}

One key aspect is that TMA usually generate large amounts of data. For example, the collection, every second, of the position for all the players of both teams in a basketball game would generate more than 20,000 positions, excluding data on speed, direction, heart rate, etc. The inspection and interpretation of these data using conventional techniques (basic statistics and charts, reporting, etc.) is therefore often difficult and tedious.

Visual Analytics [21] gives us a methodology that supports the cognitive process of data analysis and decision making by displaying information interactively. Based on different branches, such as the psychology of reasoning and perception, design aesthetics, data mining, human-computer interaction or information visualization, visual analysis eases the cognitive load in the process of analysing large volumes of data by incorporating perceptive intelligence to the process of abstract reasoning. The state of the art over TMA does not usually cover the aspects of visual inspection of results, focusing on obtaining and validating data, and its subsequent interpretation by conventional methods or simple representations. Visual analysis can greatly facilitate and accelerate the understanding of the data obtained through TMA. Even, and thanks also to the evolution of the technology needed to acquire data, interactive visual TMA can become useful not only as a tool for performance analysis a posteriori, but also as a tool for analysis and decision making in real time during the celebration of games or other sporting events.

Visual Analytics seeks to facilitate the analysis process by relying on interactive representations of data. There are several generic models of reasoning that integrate visualization and data mining interaction [7][4] and that can be tailored to specific problems such as TMA in basketball. In particular, visual analysis has been applied to fields that generate very large data volumes, such as biology, where we have data sets such as gene expression data, with tens of thousands of genes under hundreds of conditions [17][16], or that deal with space-time problems such as in [20][15]. Finally, although 
using synthetic data, visual analytics has been used to approach the analysis of time and motion in virtual environments [2].

However, our literature review suggests that the application of these models explicitly and systematically to TMA in sports problems has not been addressed. There are some basic applications of data visualization that are supplied with TMA technologies for different sports. For example, RealTrack (www.realtrackfootball.com) offers four modules covering 1) the basic plot of heart rate, 2) kinematic data monitoring, 3) calculation of positional relationships between players and their graphic representation and 4) video module that allows browsing the data collected over time. Especially in football, many similar systems to RealTrack have been developed in the last three or four years (for a review of them, see [1]). These systems offer visualization techniques that are becoming standards in the field of TMA, but these displays are usually isolated from each other, hampering the integration of knowledge and their interfaces are limited, which slows the flow of reasoning. An approach based on visual analysis would improve the cognitive ability of the athlete or coach to make decisions about training and tactics to follow, revealing additional knowledge about the problem.

\section{Goal and Requirements}

A visual analytics approach must provide the basis for an analytic discourse between the analyst and the information. This discourse is iterative, non linear and evolutive, and must facilitate the testing of different hypothesis, the addressing of the problem at different levels of abstraction, and from different points of view [21].

In the visual analytics area, presentation of results is just the last step of an iterative process that involves previous phases in which visualization (in this case, of the basketball players' performance) has a main role. In order to build a guideline of the visual analysis of the basketball game, we will identify which are the main questions that can be asked to a basketball visual TMA tool by the coach, and how to develop a model to answer them from a visual analytics point of view.

The main question a visual TMA tool for basketball answers is: Where was a player in the court at a given moment and how this position relates to the rest of the players? Specific instances of this question can generate unlimited hypotheses to be tested using an analytic discourse supported by visual analytics. For example, the hypotheses the opponent's high score is due to a small area covered in defence by my team or half court pace changes broke the opponents' zone pressure tactics are just instances of this main question. In addition, we can split the main question into several questions, and then combine them to build our list of requirements.

The following were the objectives of our proposal partially based on the requirements extracted from what a generic basketball's coach would want from an interactive visual TMA tool for the basketball game:

- If possible, the visualizations must be based on the current coaches' practice of diagrammatic representations (see figure 1)

- Handling of GPS devices. The application must be able to read files exported from a GPS device. Also, it will be necessary to transform the data from the GPS co- 


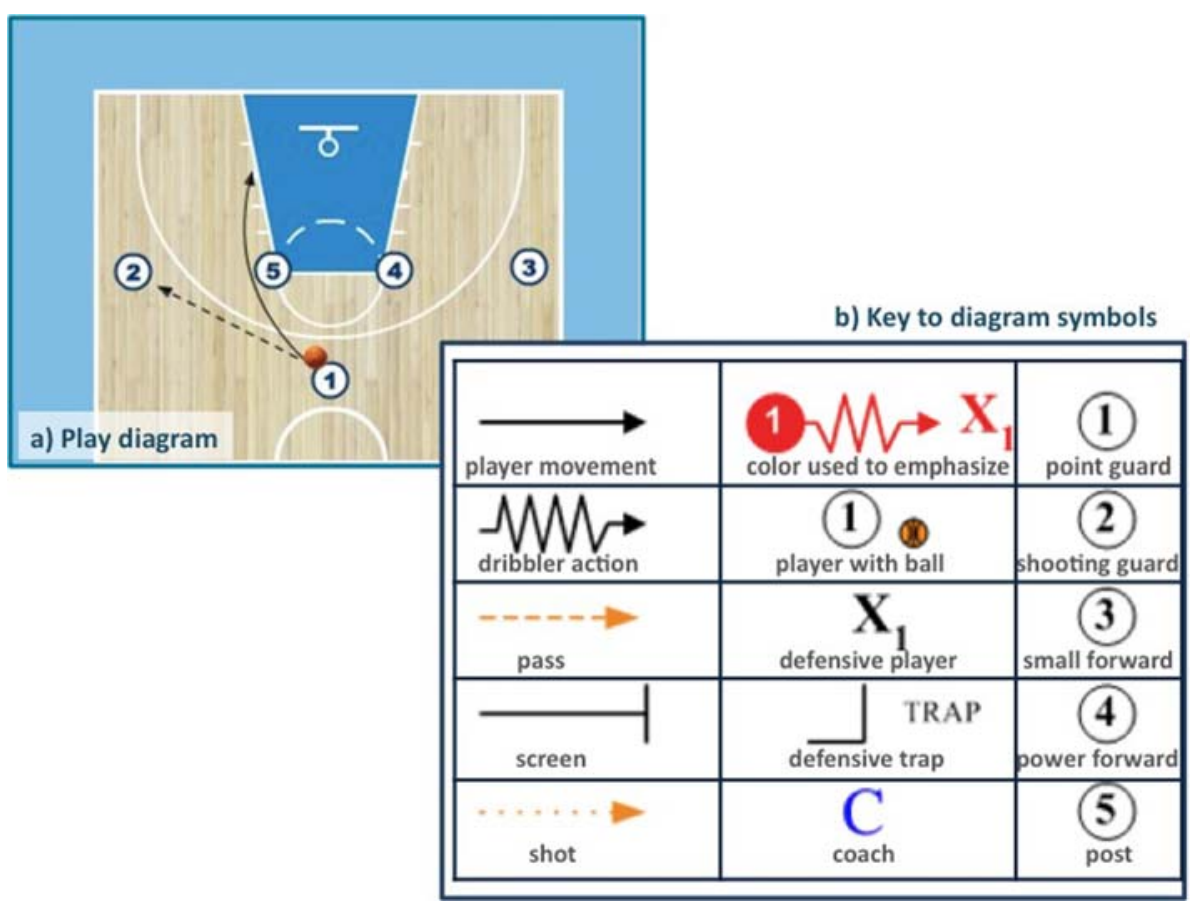

Fig. 1. a) Basketball play diagrams are the universal language of basketball coaches. By using a small set of symbols, complex play actions involving positions of the players during the duration of the play are described, i.e., a static representation of time and motion (image taken from http://coaching.fibaeurope.com). b) In the table, the basic set of symbols are presented: offensive players are depicted as numbered circles and a pass is represented as a dashed line. Thus, the diagram in a) explains a play in which the point guard passes the ball to the shooting guard and advances towards the basket, running behind the post.

ordinates (degrees: latitude, longitude) to UTM (Universal Transverse Mercator, in meters) and scale them to fit on the screen.

- Representation of position and trajectory. The position and trajectory of movement of each player at any given time of the game or exercise been analysed must be available to the user.

- Take into account the individual performance of each player: substitutions. In a basketball game there are numerous substitutions between players entering and leaving the court upon the coach's decision. As part of the players and team analysis, the tool must be able to deal with these changes of the players in court and represent them in a way.

- Time-motion analysis. The main objective of the tool is to be able to ask the tool about the position of the player at any time of the exercise or game. For that purpose, the tool should have an interactive timeline that allows the user to navigate through each player's movement history at will and to filter out events in order to focus on particular time intervals. The game situations and player actions in the 
court must be reproduced in movie mode or inspected step by step. Furthermore, regarding the TMA, the tool must be able to:

- Compute and represent areas and distances. Given that the use of spaces is very important in a sport like basketball, the tool will have to allow dynamic visualization of the area occupied between several players and the calculation and presentation of the distance between two players or a player and a point of the court at any time.

- Compute and represent kinematic variables. The system must be able to calculate and plot the kinematic variables (speed, distance travelled, time) player for statistical and analytical purposes.

- Compare players and teams. The tool should allow the visualization of the comparison of several kinematic variables of the two players or teams of users.

- Produce reports. In order to track players throughout the season, the coach should have access to a report of every player in PDF format displaying all the data and representations provided by the tool.

- Finally, the tool should incorporate automated data processing mechanisms and a highly interactive visual interface that fosters the analysis.

\section{Interactive Visual TMA of Basketball}

In this section we present and discuss several key aspects and design choices of our proposal.

\subsection{GPS data acquisition}

Once received the data from our GPS devices, we noticed that the format of the exported files were TCX, a proprietary format from the manufacturer Garmin. Thus, the first step in order to be able to visualise the data was to convert these files to the XML format. There is no standard XML format for GPS devices and we had to decide upon using one of the two most common XML schemas for geographic information, GPX (GPS eXchange Format) and KML (Keyhole Markup Language). After testing both formats, we opted for GPX, because KML was too heavy and forced us to take into account too many elements, while we wanted to obtain only the latitudes, longitudes and time of each position. In addition, GPX is a format much more widespread among GPS devices.

Next step in order to fulfil our objective was to convert geographic coordinates (latitude and longitude degrees) to UTM (meters). Finally, as mentioned previously, the converted points where scaled down to fit in the screen representation.

\subsection{Orientation of the dataset}

Once we got the coordinates of the points represented by our tool, a new problem arose that was unexpected. The court, in our representation, is oriented horizontally, however, the coordinates were obtained with the Earth globe as reference and, therefore, until their representation in the tool is shown, is not possible to comprehend the right 


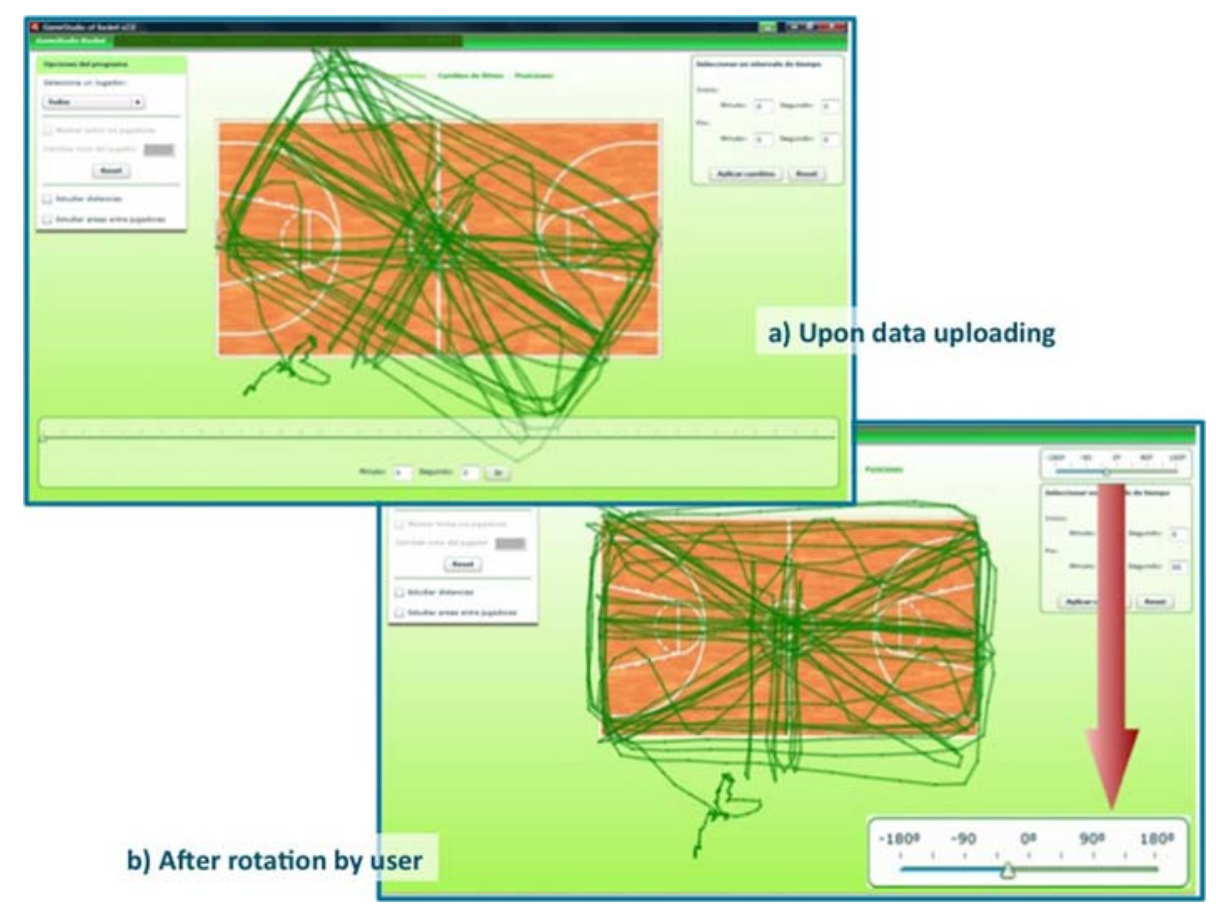

Fig. 2. a) The data acquired by the GPS devices are transformed, scaled down and represented in the visual interface. As it can be seen the representation is centred in the court thanks to the initial jump of the game; however, it is rotated in relation to the represented court. b) The user can control the orientation of the dataset to fit in the court. Once this correction is done, the coach can proceed with the rest of the analysis.

direction to use, since a point in space, without further information, does not have an orientation by itself. In this case we implemented a control for the user to orientate appropriately the representation (see figure 2).

\subsection{Motion visualization}

Figure 1 shows the set of symbols used for representing basketball players and their movements. Based on it, and not considering at this stage the representation of the ball, we decided to use the international standard representation for each player: a circle and a number within (see figure 3), while each player movement would be represented by a continuous line. Thus, the representation of the path followed by the player during the completion of a game or exercise action is a continuous line joining all the player's positions, from the first to the last one. One may argue that the result of such representation would be a concatenation of curved lines that simulate the movement of the player. However, according to the expert coaches, the typical movement of a basketball player is either straight or angled. Thus, we decided to join the positions of the player 
with straight lines (figure 2 shows in green an overview of all the positions occupied by a player during the game).

\subsection{Timeline}

For analysis purposes, a timeline control (it can be seen in figure 2.a, below the court's representation) permits the user to access the position of each player in the court at a given time by dragging a handle. We wanted to provide the tool with a simple interface, so there is also the option of moving to a particular event of the game, simply by entering the exact minute and second the coach wants to inspect in an input box.

A movie mode has also been implemented, so the coach can view the evolution of the game actions (related to a particular analysis feature, such as distances and zones, that are discussed later) for a particular period (see the movie buttons at the bottom of figure $3 . a$, for instance).

In the timeline, all the substitutions occurred during the game are also represented. To facilitate this, the timeline has a button "Enter player substitution" that gives the possibility to change the players. These changes are reflected in the course of the game animation as the timeline progresses (the player who leaves the court will disappear from the representation, while the player who enters will be visible to the user).

\subsection{Distances}

Distances between players are of particular interest for the coach. Although current GPS technology does not provide a precision to the centimetre, we decided to provide the coach with rough evidence that would be a valuable aid to tune his or her tactics. The study of distances has been designed in a simple way: the user picks as many pair of objects (a player or court point) as he or she wishes, and the distance between each pair is computed; a line from the first object to the second one is drawn, together with a label reflecting the distance in meters (see figure 3.a). Thus, 2 players (circles) or a player and a point of the court, are joined by a thin line labelled with a distance value. Once two objects have been selected for distance analysis, the lines and distance labels are updated dynamically in the movie mode.

\subsection{Areas}

In basketball, the use of space is one of the most important aspects to take into account regarding the team's performance. Therefore, we wanted to provide the coach with an option to represent the area covered at any time by 3,4 or even 5 players. This functionality aims at giving the user a clear means for understanding the use of space in a way that allows players or the coach to draw conclusions on how to play and correct mistakes at certain times of the game (see figure 3.b).

\subsection{Zones}

In conducting a full and interesting statistical study about a player we felt that a clear representation of the time that a player has been in a certain zone of the court was 


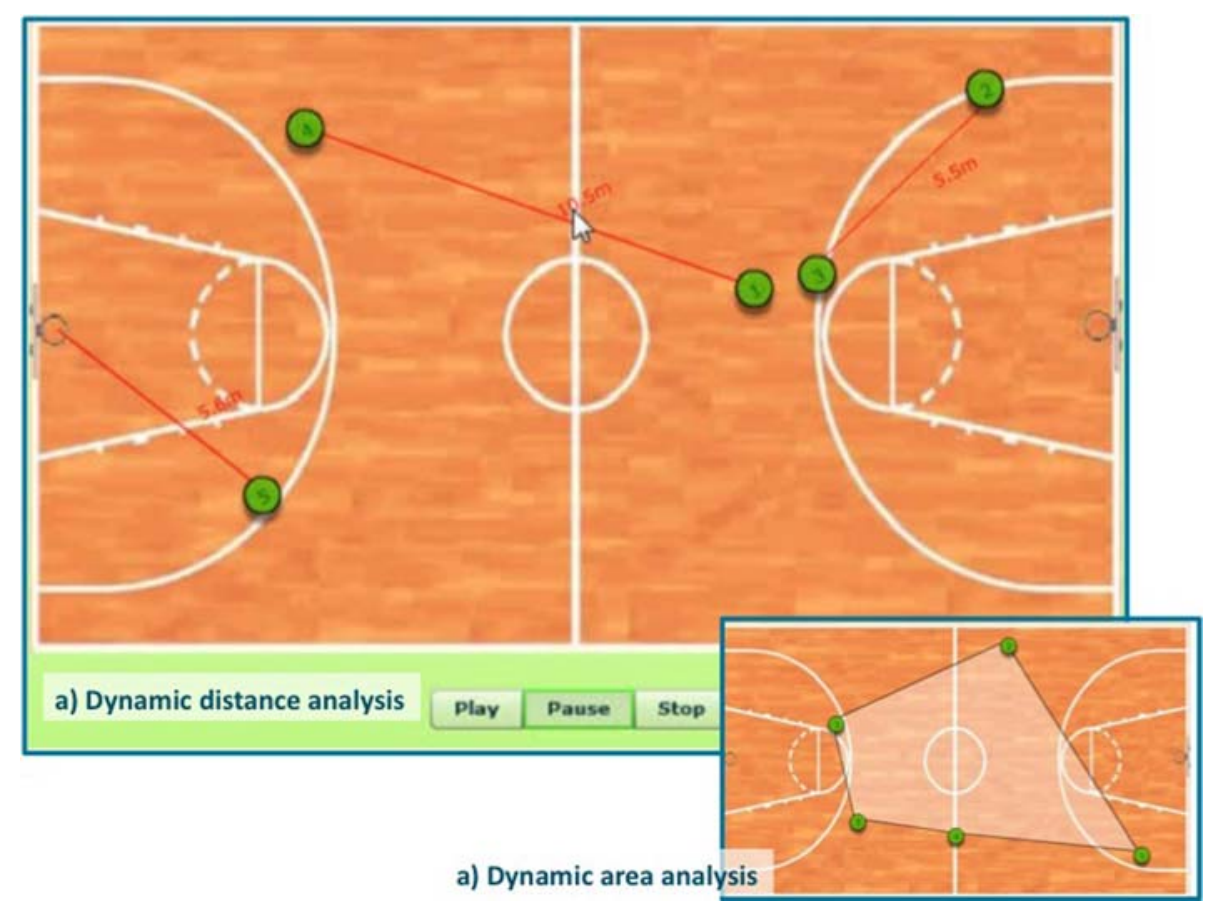

Fig. 3. a) Dynamic distance analysis. The coach has selected the five players of the team in order to study the distances among them and the basket for a particular period of time. b) Dynamic area analysis. The same study as in a) has been carried out, in this case trying to analyse how well the team are covering areas of the court, either for attack or defence, so the coach can efficiently tune the tactics or assess the performance of the team.

needed. This would allow us to know the role that a member of the team has played during the game, unravelling patterns such as the fact he or she tends to play more in a zone or simply highlight that a player is left or right handed.

\subsection{Players Comparison and Reports}

Although the application is oriented towards interactive dynamic visual analysis, we have implemented a reporting option that includes the generation of a variety of statistical charts and a numerical summary for a player or a 'players vs. player' comparison. This information is available both within the tool and as a PDF file.

\subsection{Pace changes}

Another important component of the coach's tasks is pace change analysis. Since speed is measured regarding the distance travelled in an interval of time and the change of pace is a speed change in an instant, we decided to represent the speed of player throughout the analysed period using colour coded circles, and thus, the visualization of changes 


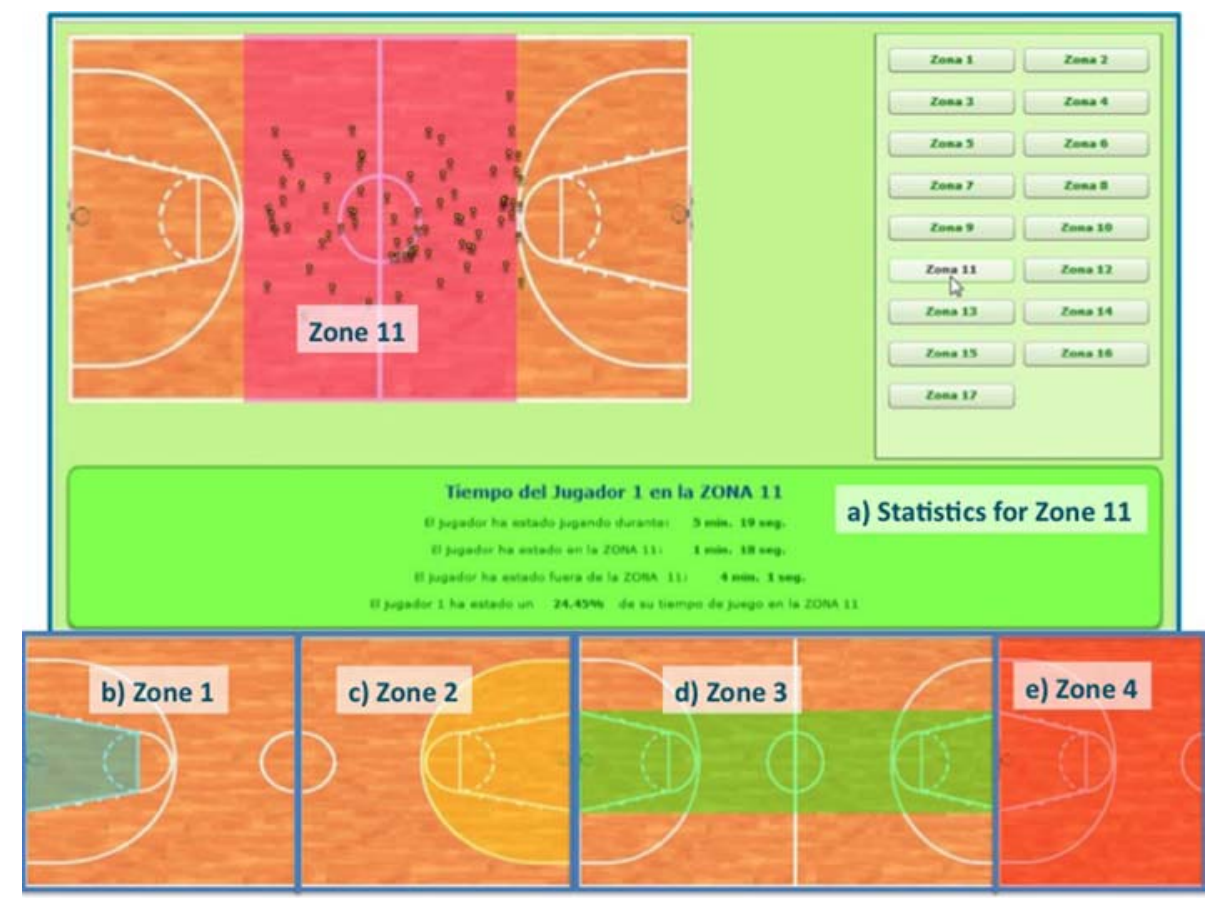

Fig.4. a) Analysis of zone 11 (midcourt zone) presence during the game for player 1. The numerical statistics are available in the dark green area. The tool has a set of 17 predefined zones, such as: b) free throw lane, c) inside the three-point line, d) basket-to-basket lane, or e) half court

of pace would be depicted as 2 consecutive circles of different colour (see figure 5.a). This method is useful when a small interval of time is analysed. However, in order to give an overview of the different paces during the whole game, we colour the player's trajectory lines using a light-to-dark scale (see figure 5.b).

\section{Conclusion and Further Work}

The development of dynamic, interactive visual solutions is a necessity in a historic moment when the generation capacity of data is huge (the accuracy of GPS devices will improve and/or combining them with other technologies such as video systems, will allow the derivation of the players' position with negligible errors) while the means of analysis have not bloomed evenly. In this regard, the recent advent of visual analytical science is very promising.

As future improvements we have considered to adapt the tool to import a file that has the complete statistics of a basketball game, so that could be visually reflected giving way to the analysis of: steals, evolution of the score of the match, points scored by each player, and so on. Moreover, and focusing on the usefulness of the tool towards the 

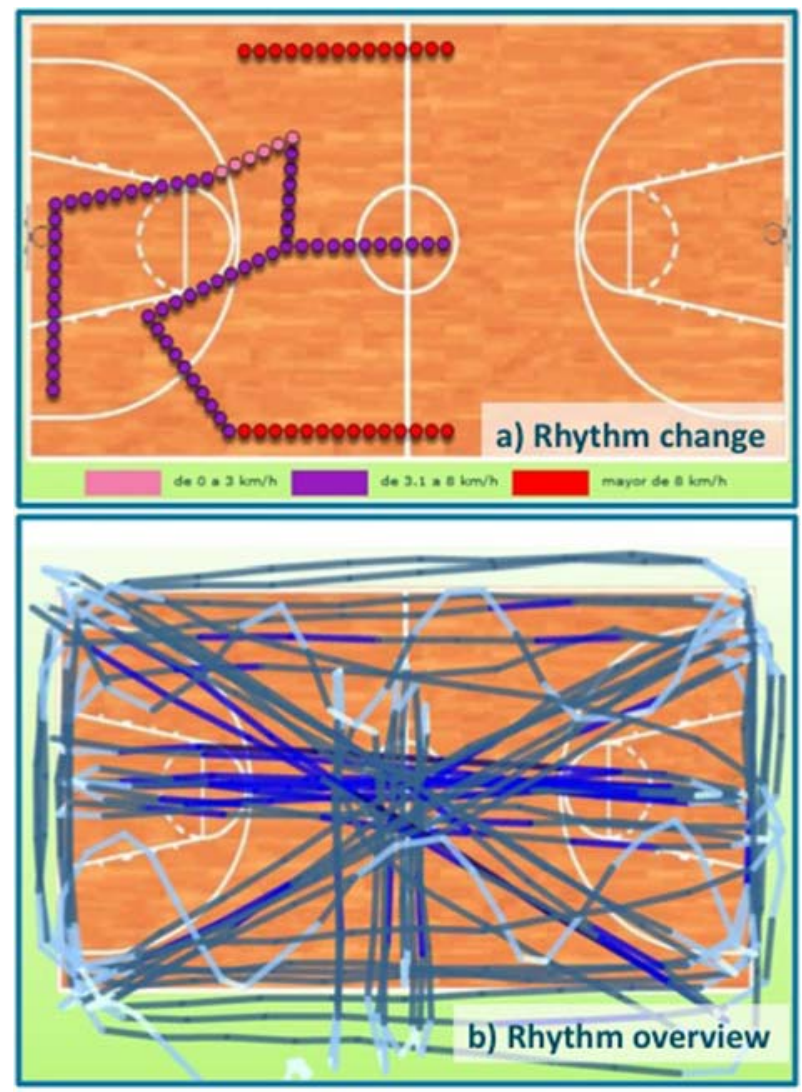

Fig.5. a) Change of pace analysis for a small interval of time (red colour means faster). b) Overview of players' pace change for the whole duration of the game (dark colour means faster). This option is more efficient for the analysis large periods of time regarding both visualization cluttering and computational load (80\% faster to compute).

physical training of the players, it would be interesting to capture and represent data in real time.

Currently, one important and missing information that has not been included yet is the representation of the ball during the basketball game. Since in this work we have focused on using GPS devices, this goal could be achieved by combining our method with the use of several cameras that capture every move on the court from different perspectives. This method would bring both more accuracy and the capture of the position of the ball.

\section{Acknowledgments}

This work was supported by the MEC (project Graccie (Consolider-Ingenio, CSD 200700067)) and by the JCyL (project GR34). 


\section{References}

1. C. Carling, J. Bloomfield, L. Nelsen, and T. Reilly. The role of motion analysis in elite soccer. Sports Med, 38(10):839, 8622008.

2. L. Chittaro, R. Ranon, and L. Ieronutti. Vu-flow: A visualization tool for analyzing navigation in virtual environments. IEEE Transactions on Visualization and Computer Graphics, Special Issue on Visual Analytics, 12(6):1475-1485, November/December 2006.

3. B. P. Dobson and J. W. L. Keogh. Methodological issues for the application of time-motion analysis research. Strength and Conditioning Journal, 29(2):48-55, 2007.

4. B. Fry. Computational Information Design. Doctoral. PhD thesis, Massachusetts Institute of Technology, 2004.

5. D. R. Gross. Time allocation: A tool for the study of cultural behavior. Ann. Rev. Anthropol., pages 519-558, 1984

6. S. Keane, T. Feilly, and N. Hughes. Analysis of work rates in gaelic football. Aust. J. Med Sci Sport., 25:100-102, 1993.

7. D. A. Keim, F. Mansmann, J. Schneidewind, H. Ziegler, and J. Thomas. Visual analytics: Scope and challenges. December 2008. Visual Data Mining: Theory, Techniques and Tools for Visual Analytics, Springer, Lecture Notes In Computer Science (lncs).

8. D. Lafontaine, M. Lamontagne, and K. Lockwood. Time-motion analysis of ice-hockey skills during games. In H. Riehle and M. V. (eds.), editors, ISBS 98: XVI International Symposium on Biomechanics in Sports, pages 481-484. International Society for Biomechanics of Sports, Konstanz, Germany., 1998.

9. F. Lothian and M. Farrally. A time-motion analysis of women's hockey. J. Hum. Movement Stud., 26:255-265, 1994.

10. S. Mayhew and H. Wegner. Time-motion analysis of professional soccer. J. Hum. Movement Stud., (11):49-52, 1985.

11. D. Mc Lean. Analysis of the physical demands of international rugby union. J. Sports Sc, 22:285-296, 1992

12. S. E. McInnes, J. S. Carslon, C. J. Jones, and M. J. McKenna. The physiological load imposed on basketball players during competitions. J. Sports Sci, 13:519-528, 1995.

13. R. Meir, A. D., and M. Forrest. Time and motion analysis of professional rugby league: A case study. Str Cond. Coach., pages 1-5, 1996.

14. M. Mohr, P. Krustrup, and J. Bangsbo. Match performance of high-standard soccer players with special reference to development of fatigue. J. Sports Sci, 21:519-528, 2003.

15. R. Santamaria and R. Theron. Treevolution: visual analysis of phylogenetic trees. Bioinformatics, 25(15):1970-1971, Aug 2009.

16. R. Santamaria, R. Theron, and L. Quintales. Bicoverlapper: a tool for bicluster visualization. Bioinformatics, 24(9):1212-1213, May 2008.

17. R. Santamaria, R. Theron, and L. Quintales. A visual analytics approach for understanding biclustering results from microarray data. BMC Bioinformatics., 9, 2008.

18. J. Taylor. Basketball: Applying time motion data to conditioning. Strength. Cond, 25 (2). J., pages 57-64, 2003.

19. P. Terrier and S. Y. How useful is satellite positioning system (gps) to track gait parameters? a review. J. Neuroengineering Rehabil., 2:28-38, 2005.

20. R. Theron. Visual analytics of paleoceanographic conditions. In Visual Analytics Science And Technology, 2006 IEEE Symposium On, pages 19-26. IEEE PRESS, 2006.

21. J. J. Thomas and K. A. Cook. A visual analytics agenda. IEEE Computer Graphics and Applications, 26:10-13, 2006.

22. W. A. M. Witte, T.H. Accuracy of non-differential gps for the determination of speed over ground. J. Biomech., 37:1891-1898, 2004. 